\title{
Outdoor Areas Lighting with LEDs - the Competition Between Scotopic Efficacy and Light Pollution
}

\author{
Irena Fryc, ${ }^{* 1}$ and Przemyslaw Tabaka ${ }^{2}$ \\ ${ }^{1}$ Bialystok University of Technology, Faculty of Electrical Engineering, Wiejska 45d, 15-351Bialystok \\ ${ }^{2}$ Lodz University of Technology, Institute of Electrical Power Engineering, Stefanowskiego 18/22, 90-924 Lodz
}

Received August 13, 2019; accepted September 27, 2019; published September, 2019

\begin{abstract}
In this paper the effect of spectral power distribution SPD of typical outdoor LED lamps on star visibility index SVI was analyzed. For those lamps the nearest color temperature CCT $[\mathrm{K}]$ and scotopic efficacy $\eta[\mathrm{lm} / \mathrm{W}]$ data are also provided. The SVI has been defined as a ratio of its scotopic flux to scotopic flux created by full moon light. The calculations show that there is a strong relation between CCT of LEDs and SVI index. Low CCT of the lamps does not significantly affect their SVI value. Unfortunately, those kind of lamps are less attractive in applications because of their low $\eta$.
\end{abstract}

Outdoor lighting is one of the most dynamically developing trends in modern lighting technology. As a result, the electricity consumption by a growing number of new lighting installations could be potentially increasing the total power consumption in an electrical power system. At the same time, it is known that the European Union member countries are obligated to reduce their total electricity consumption. Consequently, nowadays the EU countries are in the process of withdrawing high energy-consuming lamps from outdoor lighting applications. Those lamps are replaced by energy savings lamps like LEDs. Currently, high efficacy $\eta[\mathrm{lm} / \mathrm{W}]$ LED technology is becoming competitive to high-intensity discharge HID light sources, metal-halide $\mathrm{MH}$ lamps and high-pressure sodium HPS lamps. Welldesigned LED outdoor luminaires can provide the required level of illuminance with improved uniformity by using less energy, compared to HID sources. LED luminaires may also have significantly longer life $(50,000$ hours or more, compared to 15,000 to 35,000 hours for conventional lamps). Other LED advantages include: no mercury or lead content and instant start without any delay or run-up time. Typical LED lamps designed for outdoor lighting are usually assessed in terms of luminous efficacy, durability and cost of purchase and exploitation, and only little attention is paid to their potential environmental impact. This results from the fact that nowadays there are practically no legal regulations and normative requirements concerning the environmental impact of lighting. This occurs despite a well-known fact that the use of artificial lighting at night is the source of an undesirable phenomenon, which is called light

\footnotetext{
*E-mail: i.fryc@pb.edu.pl
}

pollution [1-5]. The neglect of this issue was irrelevant in the times of widespread use of incandescent sources in lighting, but in the era of modern light sources this must be necessarily considered. Contemporary lamps should be described not only by their photometric and colorimetric parameters but also by indexes describing their side effect at night [6]. There are some methods for predicting and measuring different aspects of light pollution [7-9] like glow, trespass and glare but these do not exhaust the topic because light pollution disrupts ecosystems and has adverse health effects. In addition, light pollution competes with starlight in the night sky and hereby interferes with astronomical observations even those that are performed outside big cities [10-12]. Skyglow (the scattering of light in the atmosphere at night) reduces the contrast between stars and the sky itself, making it much harder to see objects. This effect can be quantified by using the star visibility index SVI. SVI has been defined as a ratio of its scotopic light flux to scotopic light flux produced by the light of the full moon.

$$
S V I=\frac{\int_{380}^{780} S_{n(\text { lamp })}(\lambda) \cdot V^{\prime}(\lambda) d \lambda}{\int_{380}^{780} S_{\text {Moon }}(\lambda) \cdot V^{\prime}(\lambda) d \lambda}
$$

where $S_{\text {Moon }}(\lambda)$ denotes the spectral power distribution SPDs of the full moon (see Fig. 1), $V^{\prime}(\lambda)$ describes the human eye spectral sensitivity at scotopic vision (see Fig. $1)$ and $S_{n(\text { lamp })}(\lambda)$ denotes SPDs of the lamp under consideration (see Figs. 2-8). The $S_{n(\text { lamp })}(\lambda)$ must be normalized for constant luminous flux $\Phi[\mathrm{lm}]$ intensity output.

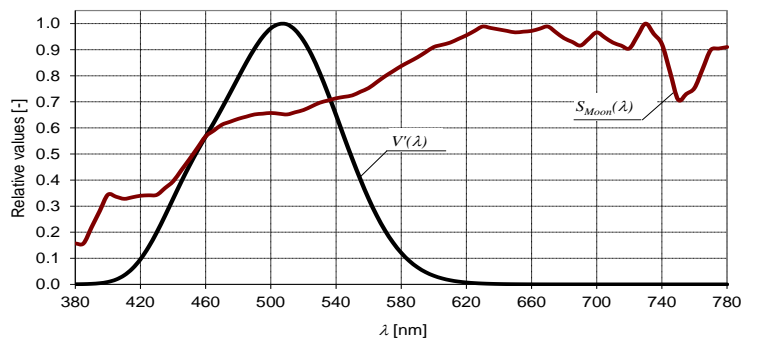

Fig. 1. Spectral power distribution of the full moon (red) and human eye scotopic sensitivity function (black). 
When the SVI value is less than 1, this is better from the star visibility point of view. Values higher than 1 mean that the conditions to see the stars are worse than when the full moon is on.

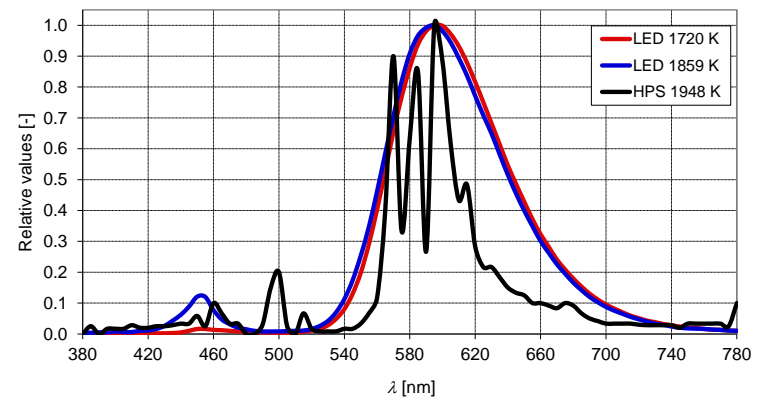

Fig. 2. The SPDs of LEDs with warm color similar to HPS lamp CCT.

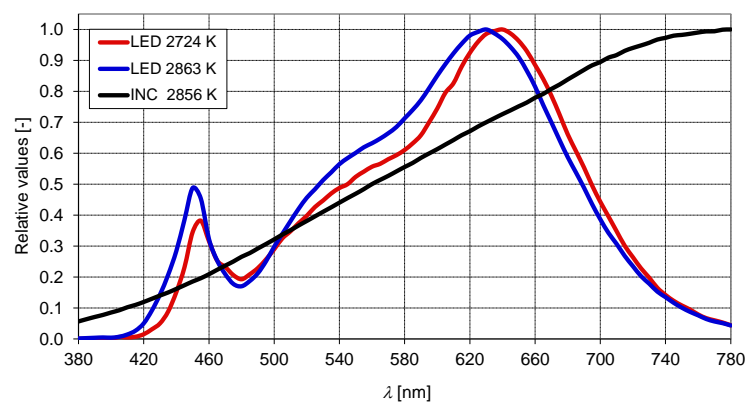

Fig. 3. The SPDs of LEDs with warm color similar to INC lamp CCT.

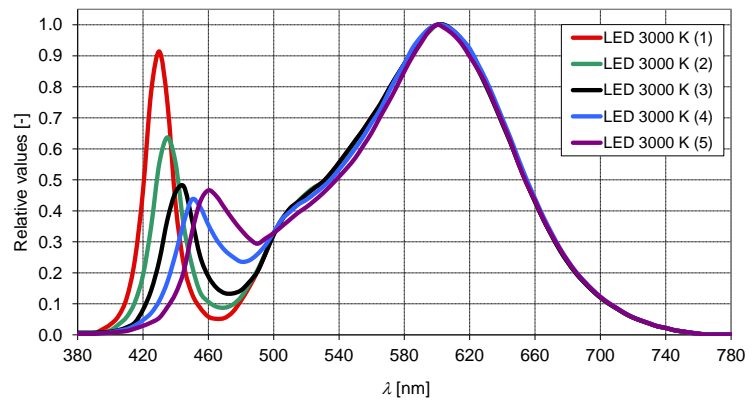

Fig. 4. The SPDs of LEDs with warm CCT $=3000 \mathrm{~K}$.

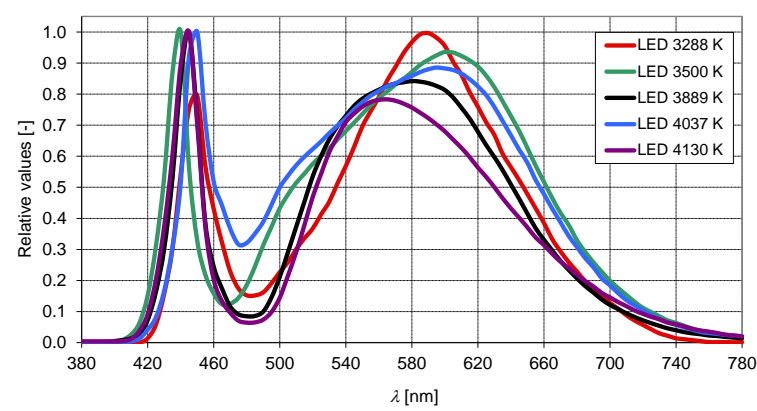

Fig. 5. The SPDs of LEDs with neutral CCT.

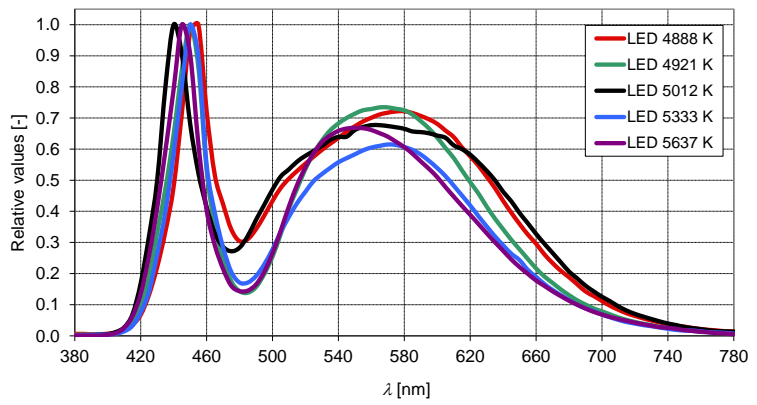

Fig. 6. The SPDs of LEDs with cold CCT.

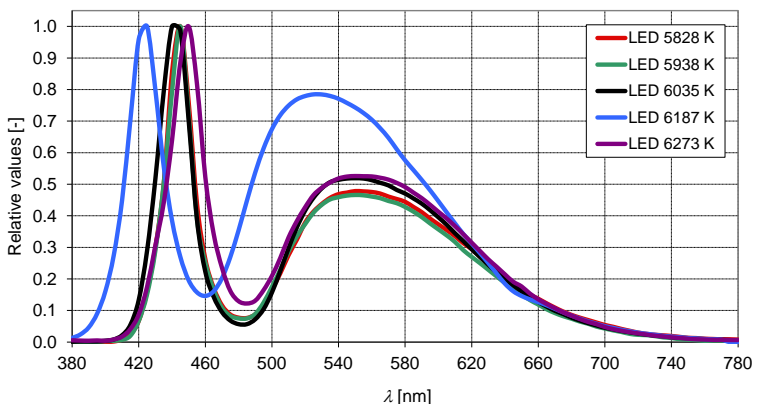

Fig. 7. The SPDs of LEDs with cold CCT.

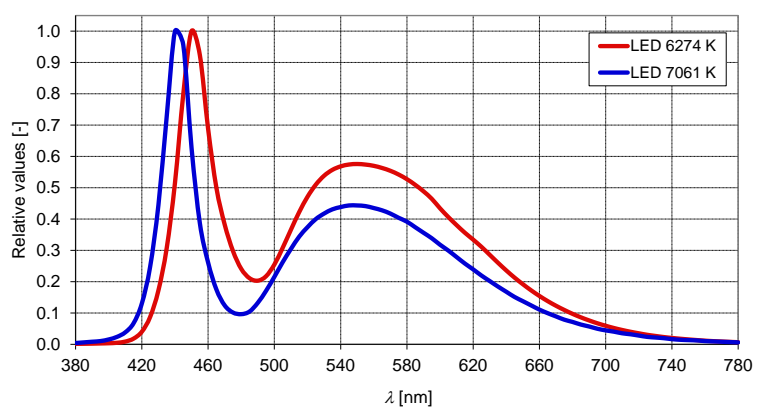

Fig. 8. The SPDs of LEDs with very cold CCT.

In contemporary outdoor lighting there is a possibility to use LEDs with any color of white light which can be described by the value of chromaticity point $x, y$ (see Fig. 9) and/or correlated color temperature CCT [K] (see Figs. 2-8). The current LED lighting product quality can vary significantly among manufacturers, so due diligence is required in their proper selection and use.

For any source of light, the scotopic efficiency $\eta$ (Fig. 10) could be defined as the ratio of its scotopic luminous flux value [lm] to optical power [W]. Is possible to see that there is a strong relation between CCT and scotopic $\eta$. The scotopic $\eta$ is increasing with CCT increasing. Cold LED lamps (with strong blue light emission) characterized by a high value of CCT have the highest value of scotopic $\eta$. For sustainable lighting design it is very important to know how different white LEDs 
(characterized by different CCT values) are affecting the side effect of night light described by the star visibility index SVI. The SVI calculations performed for 26 types of LEDs (having different SPDs and CCT) are shown in Fig. 11. For comparison, the SVI value for daylight D65 and Moon light and INC and HPS lamp are also provided in this picture.

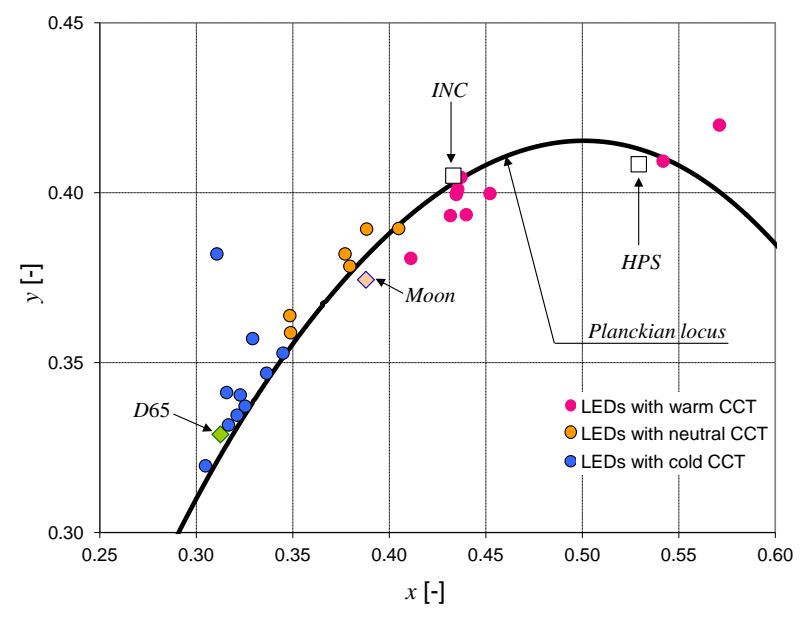

Fig. 9. The light sources chromaticity point $(x, y)$ value.

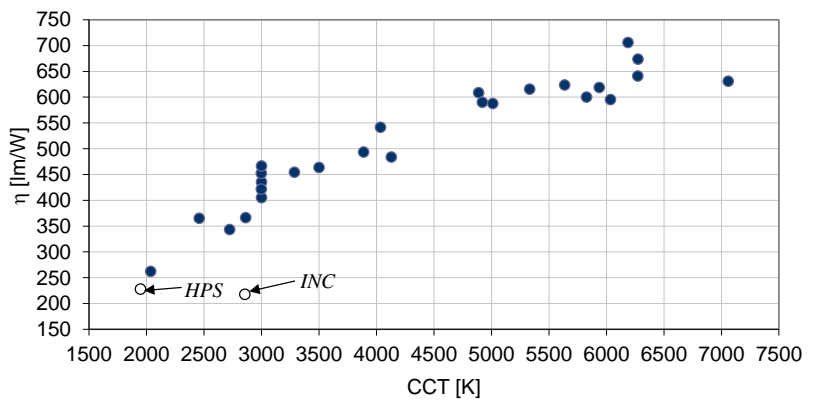

Fig. 10. The scotopic efficacy of HPS, INC and LEDs (blue dots) lamps.

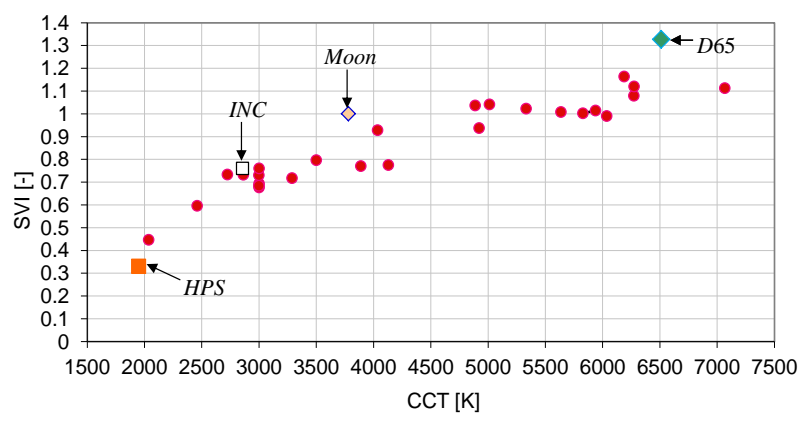

Fig. 11. The SVI versus lamp's CCT - red dots are for LEDs.

There is a strong relation between the CCT of LEDs and the value of their SVI index. For LED lamps characterized by a low value of CCT (warm light lamps) the SVI is less than 1 - which means that that kind of lamps perform well from the star visibility index point of view. For LEDs with a high value of CCT (cold lamps) the SVI index is higher than 1 . This means that when that kind of sources are used in lighting applications the star visibility will be disturbed by them. Unfortunately, lamps of this kind are very common in outdoor lighting applications because of their high scotopic lighting efficacy. Therefore, for any lighting there is always a competition between scotopic efficacy and light pollution.

Whenever only lighting scotopic efficacy is taken under consideration, cold LEDs are installed in outdoor lighting (see Fig. 10). However, from the environmental point of view warm LEDs are the best choice (see Fig. 11). Those kinds of LEDs are similar concerning the value of their SVI index to that of HPS lamps. Furthermore, the LEDs with neutral CCT have a similar STI index to that of the full moon. It seems that those kinds of LEDs may provide balance between scotopic efficiency and light pollution in outdoor lighting applications.

This work S/WE/3/2018 was supported by the Polish Ministry of Scientific Research and Higher Education research founds.

\section{References}

[1] C. Rich, T. Longcore, Ecological Consequences of Artificial Night Lighting (Island Press, Washington 2006).

[2] T. Longcore, C. Rich, Front. Ecol. Environ. 2(4), 191 (2004).

[3] K.J. Navara, R.J. Nelson, J. Pineal. Res. 43, 215 (2007).

[4] A. Bierman, Lighting Res. Technol. 44, 449 (2012).

[5] Ch.B. Luginbuhl, P.A. Boley, D.R. Davis, J. Quantitative Spectroscopy and Radiative Transfer 139, 21 (2014).

[6] M. Aube, J. Roby J, M. Kocifaj, PLOS ONE 8, e67798 (2013).

[7] J.A. Brons, J.D. Bullough, M.S. Rea, Lighting Res. Technol. 40, 201 (2008)

[8] I. Fryc, P. Tabaka, Przeglad Elektrotechniczny R93(6), 46 (2017).

[9] I. Fryc, F. Bisegna, P. Tabaka, Lighting of recreation grounds as a source of sky glow - the influence of luminaire type on this phenomenon, 1st IEEE International Conference On Environment And Electrical Engineering and 17th IEEE Industrial And Commercial Power Systems Europe (2017)

[10] Ch.B. Luginbuhl, C.E. Walker, R.J. Wainscoat, Phys. Today 62(12), 32 (2009).

[11] G.W. Lockwood, D.T. Thompson, R.D. Floyd, Publications of the Astronomical Society of the Pacific 102(650), 481 (1990).

[12] Ch.B. Luginbuhl, P.A. Boley, D.R. Davis, D.M. Duriscoe, Highlights of Astronomy 16, 765 (2014). 